\title{
Definitive 3D-CRT for clinically localized prostate cancer: modifications of the clinical target volume following a prostate MRI and the clinical benefits
}

Shinsaku Yamaguchi ${ }^{1}$, Takayuki Ohguri ${ }^{2}$, Masami Fujii' ${ }^{2}$, Katsuya Yahara ${ }^{2}$, Yoshiko Hayashida², Naohiro Fujimoto ${ }^{3}$ and Yukunori Korogi ${ }^{2}$

\begin{abstract}
Purpose: To evaluate the modifications of the tumor stage and clinical target volume following a prostate magnetic resonance imaging (MRI) to evaluate the tumor (T) staging, and the clinical benefits for prostate cancer.

Methods: A total of 410 patients with newly diagnosed and clinically localized prostate cancer were retrospectively analyzed. The patients were treated with definitive three-dimensional conformal radiotherapy (3D-CRT). In all of the patients, digital rectal examination, transrectal ultrasound, prostate biopsy and computed tomography were performed to evaluate the clinical stage. Of the 410 patients, 189 patients had undergone a prostate MRI study to evaluate the T staging, and 221 patients had not.

Results: Modification of the T stage after the prostate MRI was seen in 39 (25\%) of the 157 evaluable patients, and a modification of the risk group was made in 14 (9\%) patients. Eventually, a modification of the CTV in 3D-CRT planning was made in 13 (8\%) patients, and 10 of these had extracapsular disease. Most of the other modifications of the T staging were associated with intracapsular lesions of prostate cancer which did not change the CTV. There were no significant differences in the biological relapse-free survival between the patients with and without a prostate MRI study.

Conclusions: Modification of the CTV were recognized in only $8 \%$ of the patients, most of whom had extracapsular disease, although that of the T stage was seen in approximately one-quarter of the patients. Prostate MRI should only be selected for patients with a high probability of extracapsular involvement.
\end{abstract}

Keywords: Prostate cancer, Magnetic resonance imaging, Clinical target volume, Tumor staging, Three-dimensional conformal radiotherapy

\section{Background}

The standard imaging modality to evaluate the tumor $(\mathrm{T})$ stage of the prostate cancer is currently transrectal ultrasound (TRUS). Recently, the use of magnetic resonance imaging (MRI) has been recommended for evaluating the $\mathrm{T}$ staging in patients with prostate cancer due to its

\footnotetext{
${ }^{*}$ Correspondence: ogurieye@med.uoeh-u.ac.jp

${ }^{2}$ Department of Radiology, University of Occupational and Environmental

Health, 1-1 Iseigaoka, Yahatanishi-ku, Kitakyushu 807-8555, Japan

Full list of author information is available at the end of the article
}

high resolution (Turkbey et al. 2009; Barentsz et al. 2012; Kurhanewicz et al. 2008). However, the sensitivity and specificity of local staging with MRI vary considerably with technique and population; with rates ranging from 14 to $100 \%$ and 67 to $100 \%$, respectively.

The $\mathrm{T}$ staging of prostate cancer plays an important role in planning radiotherapy (RT), as well as for predicting the prognosis. For example, in patients with T2 or better status in the low risk group, the clinical target volume (CTV) in RT planning should include the entire prostate only, while in patients with T3 or higher stage

\section{Springer}

(c) 2015 Yamaguchi et al. This article is distributed under the terms of the Creative Commons Attribution 4.0 International License (http://creativecommons.org/licenses/by/4.0/), which permits unrestricted use, distribution, and reproduction in any medium, provided you give appropriate credit to the original author(s) and the source, provide a link to the Creative Commons license, and indicate if changes were made. 
disease, the CTV should include the prostate, extracapsular disease and seminal vesicles. Patients with T3 or higher disease are more than six times more likely to have biochemical recurrence after radical prostatectomy compared to patients with T2 or better disease (Eggener et al. 2007).

However, there are a few clinical studies have investigated whether the addition of prostate MRI for T staging changes the CTV and/or improves the clinical outcomes (Chang et al. 2014; Mullerad et al. 2004). The purpose of this study was to assess the modifications of the T staging and CTV following a prostate MRI to evaluate the $\mathrm{T}$ stage, and the clinical benefits in patients with newly diagnosed and clinically localized prostate cancer treated with definitive 3D-CRT.

\section{Methods}

\section{Patients}

From January 1998 to December 2009, 410 consecutive patients with primary prostate cancer were included in this retrospective study with the following inclusion criteria: a pathologically confirmed adenocarcinoma of the prostate, treatment using definitive 3D-CRT with a total dose of 66 Gy or more, and neither nodal nor distant metastatic disease. The following patients were excluded: those who had hormone-refractory prostate cancer and those who underwent irradiation for the whole pelvic region. One hundred and eighty-nine of the 410 patients had undergone a prostate MRI study to evaluate the $T$ staging, while the remaining 221 patients had not. The modifications of the tumor stage and CTV were evaluated in 157 (83\%) of the 189 patients who underwent the prostate MRI study, because the image reading reports of MRI, which were written by diagnostic radiologists, were not available in the remaining 32 patients at the time of the evaluation. Written informed consent for treatment was obtained from all patients.

The pretreatment evaluation included a complete history, physical examination [including digital rectal examination (DRE)], prostate biopsy with histological evaluation, laboratory studies [complete blood count, creatinine, alkaline phosphatase and initial prostatespecific antigen (iPSA)]. Table 1 shows the patients' clinical characteristics and treatments according to the use of a prostate MRI study. The risk groups were classified according to the guidelines of the National Comprehensive Cancer Network (NCCN) (2014) tumor stage. All patients had pathologically confirmed prostate adenocarcinoma, and 401 of 410 patients (98\%) received neoadjuvant androgen deprivation therapy (ADT) (median 8.2 months; range 2.1-60.3 months); adjuvant ADT was continued in $128(32 \%)$ of the 401 patients after the completion of RT (median 21.4 months; range
Table 1 The patient characteristics and treatment methods

\begin{tabular}{|c|c|c|c|}
\hline & Prostate MRI & & $p$ \\
\hline & Yes $(n=189)$ & No $(n=221)$ & \\
\hline & No. of patients (\%) & No. of patients (\%) & \\
\hline Age, years & & & 0.50 \\
\hline Median (range) & $73(57-85)$ & $74(47-84)$ & \\
\hline Tstage $^{a}$ & & & 0.82 \\
\hline $\mathrm{T} 1-2$ & $144(76)$ & $171(77)$ & \\
\hline T3-4 & $45(24)$ & $50(23)$ & \\
\hline iPSA (ng/mL) & & & 0.91 \\
\hline$<10$ & $60(32)$ & $74(33)$ & \\
\hline $10-20$ & $57(30)$ & $68(31)$ & \\
\hline$>20$ & $71(38)$ & $79(36)$ & \\
\hline Not specified & $1(0)$ & $0(0)$ & \\
\hline Gleason score & & & 0.68 \\
\hline $2-6$ & $77(41)$ & $83(38)$ & \\
\hline 7 & $65(34)$ & $82(37)$ & \\
\hline $8-10$ & $40(21)$ & $53(24)$ & \\
\hline Not specified & $7(4)$ & $3(1)$ & \\
\hline Risk groups & & & 0.48 \\
\hline Low & 24 & 36 & \\
\hline Intermediate & $60(32)$ & 72 & \\
\hline High & 85 & 97 & \\
\hline Very high & 16 & 15 & \\
\hline Not specified & 4 & 1 & \\
\hline Date of treatment & & & $<0.001$ \\
\hline 1998-2005 & $117(62)$ & $82(37)$ & \\
\hline 2006-2009 & $72(38)$ & $139(63)$ & \\
\hline ADT & & & $<0.001$ \\
\hline$<6$ months & $17(9)$ & $46(21)$ & \\
\hline$\geq 6$ months & $171(90)$ & $167(76)$ & \\
\hline None & $1(1)$ & $8(3)$ & \\
\hline Radiation dose & & & $<0.0001$ \\
\hline $66 \mathrm{~Gy}$ & $87(46)$ & $58(26)$ & \\
\hline 70 Gy & $100(53)$ & $162(74)$ & \\
\hline Other & $2(1)$ & $1(0)$ & \\
\hline Hyperthermia & & & 0.53 \\
\hline Yes & $62(33)$ & $79(36)$ & \\
\hline No & $127(67)$ & $142(64)$ & \\
\hline
\end{tabular}

$M R I$ magnetic resonance imaging, iPSA initial prostate-specific antigen, $A D T$ androgen deprivation therapy.

a T stage after prostate MRI.

2.2-41.0 months). The median total duration of neoadjuvant plus adjuvant ADT was 8.5 months in 401 patients (range 2.1-129.0 months).

\section{Prostate MRI and interpretation}

Routine prostate imaging for a work up in patients with newly diagnosed prostate carcinoma included TRUS 
and computed tomography (CT). In all 410 patients investigated in this study, TRUS and CT was performed by attending urologists, and the prostate MRI had been additionally selected to evaluate the $\mathrm{T}$ stage for some of the patients based on the decision of each individual attending urologist. Therefore, although the present study was retrospective and non-randomized in nature, the subjects included patients with and without a prostate MRI. Of the study population of 410 patients, 189 patients (46\%) had also undergone a prostate MRI study at the time of the initial diagnosis and evaluation of the prostate cancer, and 221 patients (54\%) had not undergone a prostate MRI study.

Because prostate MRI was performed after DRE, TRUS and CT, modifications, including up- or downstaging of the $\mathrm{T}$ stage and risk group after the prostate MRI studies, were retrospectively evaluated based on the subjects' medical records, including image reading reports of MRI in each case. The image reading reports for prostate MRI were written by diagnostic radiologists for each patient.

As described below, the CTV was determined according to the risk group. Therefore, changes in the targets added at upstaging or deleted at downstaging in the CTV values obtained before prostate MRI, as appropriate for the risk group before prostate MRI, and after prostate MRI, as actually performed in the patients, were evaluated.

Scans were performed on a 1.5-Tesla MRI scanner using a phased array coil. Endorectal coils were not used in this study. The entire prostate gland and seminal vesicles were covered by axial T1-weighted spin-echo imaging (T1WI; repetition time/echo time, 450-550/8.4$8.9 \mathrm{~ms}$, echo train length $=3$, and the acquisition time was 2 min and $21 \mathrm{~s}$ ) and axial T2-weighed turbospin-echo imaging (T2WI; 3,500-3,800/85-105 ms, echo train length $=17-18$, and the acquisition time was $2 \mathrm{~min}$ and $34 \mathrm{~s}$ ). These conventional images were obtained with a $4-5 \mathrm{~mm}$ slice thickness, $1.0 \mathrm{~mm}$ interslice gap, $200-250 \mathrm{~mm}$ field of view and a matrix size of $288-256 \times 256-192$. In 127 (67\%) of the patients who underwent a prostate MRI study, gadoliniumenhanced imaging was performed. In 104 (82\%) of these 127 patients, dynamic contrast-enhanced images were obtained using a 3D gradient echo sequence (LAVA; GE Healthcare, Milwaukee, WI, USA) with ultrafast image reconstruction by parallel imaging algorithms (ASSET factor, 2) in the axial plane (TR/TE 4.4-4.6/2.1 ms; flip angle, $12^{\circ}$, slice thickness, $3-5 \mathrm{~mm} ; 320-400 \mathrm{~mm}$ field of view, matrix size of $320 \times 192-256$, and the acquisition time was $26 \mathrm{~s})$.

\section{Radiation therapy}

Radiation treatment was delivered to all patients with definitive intent. All patients were treated with a 10-MV linear accelerator using three-dimensional conformal RT (3D-CRT) planning. Computed tomography-assisted 3D-CRT planning (FOCUS or Xio; CMS Japan, Tokyo, Japan) was used to determine the radiation fields in all patients. All patients were treated in the supine position and underwent a planning $\mathrm{CT}$ scan.

A clinical target volume (CTV) was determined according to the risk group as follows: low risk, CTV1 was the entire prostate alone, and CTV2 was the same as CTV1; intermediate risk, CTV1 was the entire prostate plus proximal seminal vesicles, and CTV2 was the entire prostate; high risk, CTV1 was the entire prostate, gross extracapsular disease plus proximal seminal vesicles, and CTV2 was the entire prostate plus gross extracapsular disease and very high risk, CTV1 was the entire prostate, gross extracapsular disease plus the entire seminal vesicles, and CTV2 was the entire prostate and gross extracapsular disease. CT-MRI image fusion was not applied for the 3D-CRT planning, although prostate MRI was used to evaluate the $\mathrm{T}$ staging. Contouring of gross extracapsular disease was referred based on the prostate MRI.

The total planning dose was 66 Gy $(\mathrm{n}=145), 70$ Gy $(\mathrm{n}=262), 72 \mathrm{~Gy}(\mathrm{n}=1), 74 \mathrm{~Gy}(\mathrm{n}=1)$ or $76 \mathrm{~Gy}(\mathrm{n}=1)$, and the fraction was 2.0 Gy once a day (five times/week) (1). The planning target volume (PTV) was delineated by contouring a CTV1 with a PTV1 margin of $1.2-1.7 \mathrm{~cm}$ during the initial 46 Gy in 23 fractions, and CTV2 with a PTV2 margin of $0.7-1.2 \mathrm{~cm}$ during the subsequent $20-30$ Gy in $10-15$ fractions. The beams were shaped using a multileaf collimator. All patients were treated with 3D conformational or seven-field conformal radiation with an isocentric technique.

One hundred and forty-one (34\%) of the 410 patients were also treated with pelvic regional hyperthermia during 3D-CRT to improve the antitumor effects of RT. Hyperthermia was applied after irradiation once a week for radio-sensitization. An 8-MHz radiofrequency (RF)capacitive regional hyperthermia system (Thermotron RF-8; Yamamoto Vinita, Osaka, Japan) was used (Abe et al. 1986).

\section{Follow-up}

The length of follow-up was calculated from the date of the start of RT. The patients were followed-up at 1 to 3-month intervals during the first year, and at 3 to 6-month intervals thereafter. PSA measurement and assessments of the gastrointestinal and genitourinary morbidity were performed at each follow-up visit. Biochemical failure was defined according to the Phoenix definition (Roach et al. 2006). The clinical relapse-free survival (cRFS) was defined as the rate of freedom from 
local failure, regional failure and distant metastasis. The presence of bone metastasis was confirmed by bone scintigraphy, computed tomography or MR imaging. Soft tissue metastasis was confirmed by computed tomography or MR imaging.

\section{Statistical analysis}

The Chi square tests and Mann-Whitney $U$ tests were used to assess the baseline imbalances between the patients with and without prostate MRI. The KaplanMeier method was used to calculate the outcomes for the biochemical relapse-free survival (bRFS) and cRFS. The time period was calculated as beginning at the start of definitive 3D-CRT. To identify prognostic factors for the bRFS, univariate analyses were performed. The log-rank test was used for statistical comparisons between groups. Multivariate analyses using the Cox proportional-hazards model were also performed to determine the bRFS. Fisher's exact tests were performed to evaluate the differences in the patterns of first failure between the patients with and without prostate MRI.

\section{Results}

Table 2 shows the results for the modifications of the $\mathrm{T}$ stage, risk group and CTV in the 3D-CRT planning following prostate MRI study. Upstaging of the T stage was identified in $33(21 \%)$ of the 157 patients, while downstaging was observed in six (8\%) patients. Meanwhile, upstaging of the risk group after prostate MRI occurred in 10 patients, whereas downstaging was noted in four patients. Modifications of the CTV values were made in 11 patients with upstaging and two patients with downstaging after prostate MRI. Changes in the targets for the CTV, as added at upstaging or deleted at downstaging, are listed in Table 2. Table 3 shows the relationships among the risk groups classified according to the Prostate Cancer Risk Stratification (ProCaRS) risk group before prostate MRI, the upstaging of the $\mathrm{T}$ stage or NCCN risk group after prostate MRI, and the changes in the CTV after prostate MRI (Rodrigues et al. 2013). Eight (73\%) of 11 patients with modifications of the CTV after prostate MRI who demonstrated upstaging were associated with the ProCaRS High-intermediate risk, High-risk or Extreme-risk groups before prostate MRI.

Table 2 A summary of the modifications for the T stage, risk group and CTV following prostate MRI studies in 157 evaluable patients

\begin{tabular}{|c|c|c|c|}
\hline & $\begin{array}{l}\text { No. of } \\
\text { patients (\%) }\end{array}$ & $\begin{array}{l}\text { No. of patients with modifications } \\
\text { of the risk group after prostate MRI (\%) } \\
\text { (change of risk) }\end{array}$ & $\begin{array}{l}\text { No. of patients with modifications of the CTV in 3D-CRT } \\
\text { planning based on the prostate MRI findings (targets } \\
\text { added at upstaging or deleted at downstaging) }\end{array}$ \\
\hline No change & $118(75)$ & - & - \\
\hline Upstaging & $33(21)$ & $10(6)$ & $11(7)$ \\
\hline T1c to T2a & 10 & 0 & 0 \\
\hline $\mathrm{T} 1 \mathrm{c}$ to $\mathrm{T} 2 \mathrm{~b}$ & 10 & $1(\mathrm{~L} \rightarrow \mathrm{IM})$ & 1 (pSV in CTV1) \\
\hline T1c to T2c & 3 & $2(\mathrm{~L} \rightarrow \mathrm{IM})$ & $2(p S V$ in $C T V 1)$ \\
\hline T1с to Т3a & 2 & $1(\mathrm{~L} \rightarrow \mathrm{H})$ & 1 (pSV and GED in CTV1, GED in CTV2) \\
\hline T2a to $T 3 a$ & 1 & 0 & 1 (pSV and GED in CTV1, GED in CTV2) \\
\hline T2a to $T 3 b$ & 2 & $2(\mathrm{H} \rightarrow \mathrm{VH})$ & 2 (eSV and GED in CTV1, GED in CTV2) \\
\hline T2b to T2c & 1 & 0 & 0 \\
\hline T2b to T3a & 1 & $1(\mathrm{IM} \rightarrow \mathrm{H})$ & 1 (GED in CTV1, CED in CTV2) \\
\hline $\mathrm{T} 2 \mathrm{~b}$ to $\mathrm{T} 3 \mathrm{~b}$ & 1 & $1(\mathrm{H} \rightarrow \mathrm{VH})$ & 1 (eSV and GED in CTV1, GED in CTV2) \\
\hline T3a to T4 & 2 & $2(\mathrm{H} \rightarrow \mathrm{VH})$ & 2 (eSV and GED in CTV1, GED in CTV2) \\
\hline Downstaging & $6(4)$ & $4(3)$ & $2(1)$ \\
\hline T3a to T2a & 1 & $1(H \rightarrow I M)$ & $0^{\mathrm{a}}$ \\
\hline T3a to T2b & 2 & $1(H \rightarrow I M)$ & $0^{\mathrm{a}}$ \\
\hline T3a to T2c & 1 & 0 & 0 \\
\hline T3b to T2c & 1 & $1(\mathrm{VH} \rightarrow \mathrm{H})$ & $1^{\mathrm{a}}(\mathrm{eSV}$ in CTV1) \\
\hline T4 to T2a & 1 & $1(\mathrm{VH} \rightarrow \mathrm{H})$ & $1^{\mathrm{a}}(\mathrm{eSV}$ in CTV1) \\
\hline
\end{tabular}

$L$ low risk, IM intermediate risk, $H$ high risk, $V H$ very high risk, $p S V$ proximal seminal vesicles, eSV entire seminal vesicles, GED gross extracapsular disease, $C T V$ clinical target volume.

a A contour of the GED could not be depicted, because the GED confirmed or suspected in TRUS was not recognized in the prostate MRI study or by CT. 
Table 3 Upstaging and changes in the CTV among the risk groups after prostate MRI according to the Prostate Cancer Risk Stratification (ProCaRS) risk stratification system

\begin{tabular}{llll}
\hline $\begin{array}{l}\text { ProCaRS } \mathbf{6} \text { categories }^{\mathbf{a}} \\
\text { before prostate MRI }\end{array}$ & $\begin{array}{l}\text { Upstaging of the T stage } \\
\text { after prostate MRI }(\mathbf{n}=\mathbf{3 3})(\%)\end{array}$ & $\begin{array}{l}\text { Upstaging of the NCCN } \\
\text { risk group after prostate } \\
\text { MRI (n=10) (\%) }\end{array}$ & $\begin{array}{l}\text { Modifications of the CTV } \\
\text { in 3D-CRT planning based on the } \\
\text { prostate MRI findings (n= 11) (\%) }\end{array}$ \\
\hline Very low-risk & $1(3)$ & $1(10)$ & $1(9)$ \\
Low-risk & $3(9)$ & $1(10)$ & $1(9)$ \\
Low intermediate-risk & $8(24)$ & $1(10)$ & $1(9)$ \\
High intermediate-risk & $7(21)$ & $2(20)$ & $2(18)$ \\
High-risk & $4(12)$ & $3(30)$ & $4(36)$ \\
Extreme-risk & $10(30)$ & $2(20)$ & $2(18)$ \\
\hline
\end{tabular}

Very low-risk:T1-T2a AND PSA $\leqq 6 \mathrm{ng} / \mathrm{ml}$ AND Gleason score $\leqq 6$. Low-risk: T1-T2a AND PSA >6 AND PSA $\leqq 10 \mathrm{ng} / \mathrm{ml}$ AND Gleason score $\leqq 6$. Low intermediate-risk: T1-T2 AND PSA $\leqq 20 \mathrm{mg} / \mathrm{ml}$ AND [PSA $\leqq 10 \mathrm{ng} / \mathrm{ml}$ OR (PSA $>10 \mathrm{ng} / \mathrm{ml}$ AND \{T1-T2a OR Gleason $\leqq 6\})]$. High intermediate-risk:T1-T2 AND PSA $\leqq 20 \mathrm{mg} / \mathrm{ml}$ AND [PSA $>10 \mathrm{ng} / \mathrm{ml}$ AND (T2b/c OR Gleason 7)]. High-risk: [T3-T4 OR (PSA >20 ng/ml AND PSA <30 ng/ml) OR Gleason 8-10] AND \% cores <87.5\%. Extreme-risk: [(T3-T4 OR Gleason 8-10 OR PSA > $20 \mathrm{ng} / \mathrm{ml})$ ] AND (PSA $\geqq 30 \mathrm{ng} / \mathrm{ml}$ OR \% cores $\geqq 87.5 \%$ ).

a Rodrigues et al. (2013).

The median follow-up in all patients was 67.3 months (range 0.1-161.6 months), while that in the 189 patients with a prostate MRI study was 79.1 months (range 0.1-161.6 months), and that in the 221 patients without a prostate MRI study was 68.8 months (range 0.4157.8 months). Table 4 shows the prognostic factors in the univariate analyses for the bRFS. The 5-year and the 3-year bRFS were 86 and $89 \%$ in the patients with a prostate MRI study and 90 and $92 \%$ in the patients without a prostate MRI study, respectively, and these values were not significantly different (Figure 1 ). The 5-year and 3-year cRFS were 96 and $98 \%$ in the patients with a prostate MRI study, and 97 and $99 \%$ in the patients without a prostate MRI study, respectively; there was also no significant difference in these values. In the multivariate analyses for the bRFS, the Gleason score was found to be a statistically significant factor, while the addition of the prostate MRI was not a predictive factor (Table 4). In the univariate subset analyses, among 315 patients with T1-2 disease, a prostate MRI study was not a significant predictor of the bRFS. It was also not a significant predictor among the 95 patients with T3-4 disease.

Clinical failure and biochemical failure had occurred in 11 and 35 patients who underwent a prostate MRI study, and in 6 and 26 patients without a prostate MRI study, respectively; there were no significant differences between the groups. The patterns of first failure after the 3D-CRT are shown in Table 5; no significant differences were seen between the patients with or without a prostate MRI study. Salvage ADT was performed after a biochemical failure in 35 (19\%) patients with a prostate MRI study and 26 (12\%) patients without a prostate MRI study. Death from prostate cancer was seen in two (1\%) patients who underwent a prostate MRI and in three (1\%) patients without a prostate MRI.

\section{Discussion}

The current study is to assess the benefits, in terms of the tumor staging, CTV and clinical outcomes, of adding prostate MRI examinations to evaluate the $T$ stage in patients with localized prostate cancer treated with 3D-CRT. Although the current study was retrospective and non-randomized in nature, the findings were obtained from a large cohort of patients treated at a single institution. With its excellent soft-tissue resolution, MRI of the prostate clearly depicts the prostate's zonal anatomy and facilitates prostate cancer localization and staging (Mullerad et al. 2004; Graser et al. 2007; Yu et al. 1997; Engelbrecht et al. 2002). MRI has been shown to contribute significant incremental value to clinical variables in the prediction of the clinical $\mathrm{T}$ stage, especially the existence of extracapsular extension and/or seminal vesicle invasion, and to significantly improve treatment planning (Mullerad et al. 2004; Sala et al. 2006; Wang et al. 2004). However, some previous studies also demonstrate that the accuracies of the $\mathrm{T}$ staging of prostate cancer between MRI and TRUS were comparable (Rifkin et al. 1990; Presti et al. 1996; Ekici et al. 1999). In the current study, modifications of the CTV for treatment planning with 3D-CRT based on the results of prostate MRI were made in only $8 \%$ of the patients. Furthermore, the addition of prostate MRI to TRUS did not improve the bRFS after 3D-CRT in the patients with clinically localized prostate cancer. We therefore confirmed that the clinical benefits of adding prostate MRI to TRUS to determine the $\mathrm{T}$ stage are limited in patients with localized prostate cancer treated with 3D-CRT.

As mentioned in the "Background", the CTV in RT planning for prostate cancer depends on the risk group; for the low risk group, the risk of seminal vesicle involvement is $<5 \%$; therefore, the CTV should be restricted 
Table 4 Results of the univariate and multivariate analyses of factors associated with the bRFS after definitive radiotherapy

\begin{tabular}{|c|c|c|c|c|c|}
\hline & $\mathrm{n}$ & Univariate & & Multivariate $^{\mathrm{f}}$ & \\
\hline & & 5-year (\%) & $p$ & OR $(95 \% \mathrm{Cl})$ & $p$ \\
\hline Age (years) & & & $<0.01$ & $1.71(0.99-2.99)$ & 0.06 \\
\hline$<70$ & 130 & 82 & & & \\
\hline$>71$ & 280 & 90 & & & \\
\hline T stage $\mathrm{e}^{\mathrm{a}}$ & & & $<0.01$ & $1.88(0.96-3.71)$ & 0.07 \\
\hline $\mathrm{T} 1-2$ & 315 & 92 & & & \\
\hline T3-4 & 95 & 74 & & & \\
\hline iPSA $(n g / m L)^{b}$ & & & $<0.01$ & $1.76(0.83-3.72)$ & 0.14 \\
\hline$\leqq 20$ & 258 & 91 & & & \\
\hline$>20$ & 151 & 82 & & & \\
\hline Gleason score ${ }^{c}$ & & & $<0.01$ & $3.06(1.60-5.81)$ & $<0.001$ \\
\hline $2-7$ & 308 & 91 & & & \\
\hline $8-10$ & 92 & 75 & & & \\
\hline Risk group ${ }^{d}$ & & & $<0.01$ & $1.30(0.47-3.63)$ & 0.62 \\
\hline $\begin{array}{l}\text { Low-intermediate } \\
\text { risk }\end{array}$ & 191 & 92 & & & \\
\hline High-very high risk & 214 & 83 & & & \\
\hline Date of treatment & & & 0.11 & $1.41(0.66-3.02)$ & 0.37 \\
\hline 1998-2005 & 199 & 90 & & & \\
\hline 2006-2009 & 211 & 86 & & & \\
\hline ADT (months) ${ }^{e}$ & & & 0.45 & $1.07(0.47-2.44)$ & 0.87 \\
\hline$<6$ & 63 & 91 & & & \\
\hline$\geqq 6$ & 338 & 88 & & & \\
\hline Radiation dose & & & 0.06 & $1.51(0.67-3.40)$ & 0.32 \\
\hline$<70$ Gy & 145 & 85 & & & \\
\hline$\geqq 70 \mathrm{~Gy}$ & 265 & 92 & & & \\
\hline Hyperthermia & & & 0.18 & $1.21(0.65-2.26)$ & 0.54 \\
\hline Yes & 141 & 86 & & & \\
\hline No & 269 & 88 & & & \\
\hline Prostate MRI study & & & 0.33 & $1.57(0.89-2.77)$ & 0.12 \\
\hline Yes & 189 & 86 & & & \\
\hline No & 221 & 90 & & & \\
\hline
\end{tabular}

bRFS biochemical relapse-free survival, EBRT external beam radiation therapy, $M R I$ magnetic resonance imaging, $O R$ odds ratio, $\mathrm{Cl}$ confidence interval.

a T stage after prostate MRI.

b Excluding 1 patient with unknown iPSA.

c Excluding 10 patients with unknown Gleason scores.

${ }^{d}$ Excluding 5 patients with an unknown risk group status.

e Excluding 9 patients without ADT.

f The 388 evaluable patients with complete factors.

to the prostate only. In contrast, for the intermediate to high risk group, the risk of seminal vesicle involvement is higher (over 15\%), so the proximal seminal vesicles should be included in the CTV, and for patients with proven seminal vesicle involvement (T3b), the entire seminal vesicle should be included in the CTV (Boehmer

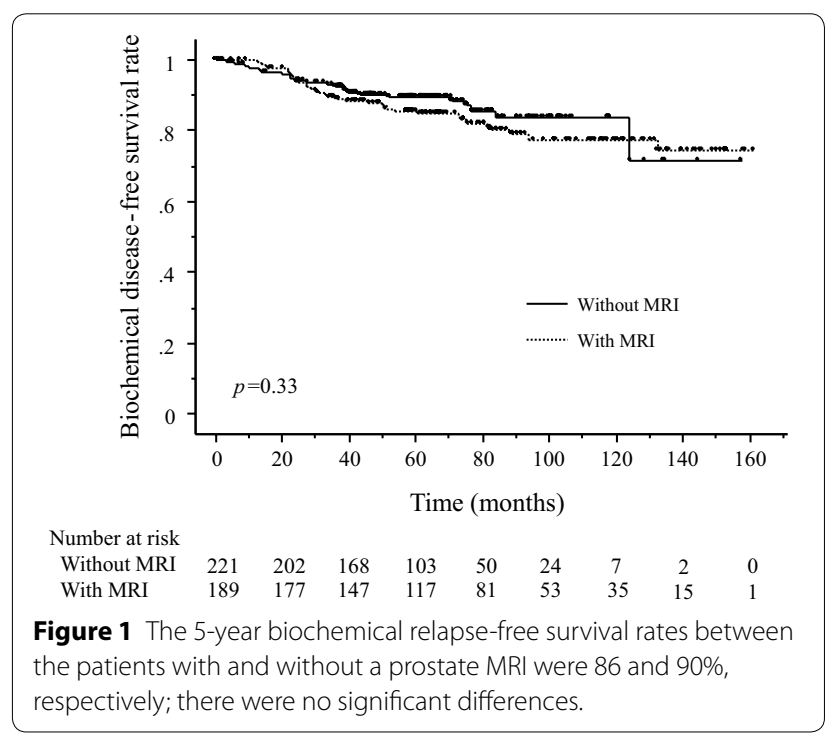

et al. 2006; Hayden et al. 2010). In addition, when gross extracapsular disease is recognized under the CTV, a margin of $2-5 \mathrm{~mm}$ (excluding the rectum) should be considered (Boehmer et al. 2006; Hayden et al. 2010). In the current study, a modification of the $T$ stage after the prostate MRI was seen in 39 (25\%) patients; however, there was a modification of the risk group in only 14 (9\%) patients, and eventually, a modification of the CTV in 3D-CRT planning only 13 (8\%) patients. Gross extracapsular disease was newly found by the prostate MRI in only six (4\%) patients, and all of them had a modification of the CTV in 3D-CRT-planing. Most of the other modifications detected were associated with intracapsular lesions of prostate cancer which did not change the risk group or the CTV. We supposed that these factors may explain why the addition of prostate MRI did not correlate with any improvement of the clinical outcomes.

Prostate MRI adds significantly to the cost of treatment when used routinely. Jager et al. (2000) reported the results of a decision analysis for the appropriate use of MRI for the preoperative staging of prostate cancer, and concluded that MRI staging is cost-effective for patients with a moderate or high prior probability of extracapsular disease. The clinical and pathological parameters predicting extracapsular disease in patients undergoing a radical prostatectomy for clinically localized prostate cancer have been reported in previous studies, and include a smaller prostate volume and positive cores for malignancy from both lobes after prostate biopsy (Sfoungaristos and Perimenis 2012). In the current study, 10 of the 13 patients with a modification of the CTV based on the prostate MRI had gross extracapsular disease. In addition, the modification of the CTV mainly occurred 
Table 5 The patterns of first failure after definitive radiotherapy

\begin{tabular}{|c|c|c|c|}
\hline & \multicolumn{2}{|l|}{ Prostate MRI } & \multirow[t]{3}{*}{$p$} \\
\hline & Yes $(n=189)$ & No $(n=221)$ & \\
\hline & No. of patients (\%) & No. of patients (\%) & \\
\hline Biochemical failure alone & $24(13)$ & $20(9)$ & 0.26 \\
\hline Clinical failure & $9(5)$ & $4(2)$ & 0.10 \\
\hline Primary alone & 1 & 0 & \\
\hline Regional lymph node alone & 2 & 0 & \\
\hline Distant metastasis alone & 4 & 3 & \\
\hline Primary, regional and distant metastasis & 1 & 0 & \\
\hline Regional lymph node and distant metastasis & 1 & 1 & \\
\hline
\end{tabular}

in the patients with high-intermediate risk, high-risk or extreme-risk group on ProCaRS before prostate MRI. Therefore, we also believed that MRI should be performed for select patients with a high probability of extracapsular disease.

Although phased-array MRI on a $1.5 \mathrm{~T}$ scanner was used in the current study, some previous studies indicated that endorectal coil MRI significantly improved the $\mathrm{T}$ staging of prostate cancer (Futterer et al. 2007; Heijmink et al. 2007). Futterer et al. (2007) reported a comparison of the local staging accuracy of pelvic phased-array coil alone versus integrated endorectal-pelvic phased-array coils on a $1.5 \mathrm{~T}$ scanner, and concluded that the use of endorectal-pelvic phased array coils resulted in significant improvement of extracapsular extension accuracy and specificity. In addition, some studies have reported that the $3.0 \mathrm{~T}$ phased-array MRI is equivalent to the $1.5 \mathrm{~T}$ endorectal MRI in evaluating the local staging accuracy for prostate cancer, without a significant loss of imaging quality (Park et al. 2007; Torricelli et al. 2006). Multiparametric MRI, such as anatomic T2-weighted imaging with MR spectroscopic imaging, dynamic contrast-enhanced MR imaging, and diffusion-weighted imaging, also demonstrated great interest in the evaluation of the prostate cancer (Sciarra et al. 2011). Further studies of pelvic MRI using these advanced techniques are therefore needed to evaluate the relationships between the $T$ staging, especially for extracapsular disease, and the clinical benefits.

Our study is associated with several potential limitations. First, due to the fact that the study was retrospective, the possibility of a selection bias with respect to the prognostic factors cannot be ruled out, although we performed both univariate and multivariate analyses of the bRFS. Therefore, in the future, prospective studies will be needed to investigate the actual clinical benefits of prostate MRI. Second, in the current study, modifications of the duration of ADT, based on the results of the addition of prostate MRI, could not be evaluated, because the treatment policy for ADT was variable among the attending urologists, although period of ADT is basically selected depending on the risk group. We believe that the results of prostate MRI would lead to limited or not modification of the period of ADT, because only 14 (9\%) patients had a change in risk group after prostate MRI. The duration of ADT in patients who underwent prostate MRI was longer than those in patients who did not, which may have influenced the results of the bRFS, although both the univariate and multivariate analyses demonstrated that the duration of ADT was not a significant factor on the bDFS. Third, hyperthermia was added to 3D-CRT to obtain radio-sensitization in select patients due to the lower total dose of 3D-CRT, which may have led to a bias in the study. Several phase II clinical trials on radiotherapy combined with hyperthermia showed promising results in patients with prostate cancer (Maluta et al. 2007; Anscher et al. 1992; Van Vulpen et al. 2004). However, in the current study, the addition of hyperthermia was not a significant factor on the bRFS. Fourth, patients who received early treatment were more likely to have undergone prostate MRI. Therefore, the follow-up duration in the patients who underwent prostate MRI was longer than that in the patients who did not. The difference in the follow-up duration may have had an impact on the results of the bRFS, although the date of the treatment was not a significant factor in the univariate/multivariate analyses on the bRFS (Table 4).

In summary, modifications of the CTV for treatment planning with 3D-CRT based on the results of prostate MRI were identified in only $8 \%$ of the patients in the present study, most of whom had extracapsular disease. In contrast, modification of the $\mathrm{T}$ stage after prostate MRI was observed in approximately one-quarter of the patients, and the addition of prostate MRI was not associated with any improvements in outcomes after 3D-CRT. Therefore, the clinical benefits of adding prostate MRI to evaluate the $\mathrm{T}$ stage may be limited in patients with 
localized prostate cancer treated with 3D-CRT. Prostate MRI should not be performed in every patient with clinically localized prostate carcinoma, but is recommended for the patients with a high prior probability of extracapsular disease.

\section{Authors' contributions}

YS collected and analyzed data, and drafted the manuscript; TO analyzed data and drafted the manuscript; MF collected and analyzed data; KY contributed to data analysis; $\mathrm{YH}$ contributed to data analysis; NF revised the manuscript; YK revised the manuscript. All authors read and approved the final manuscript.

\section{Author details}

${ }^{1}$ Department of Radiology, Kitakyushu General Hospital, Kitakyushu, Japan. 2 Department of Radiology, University of Occupational and Environmental Health, 1-1 Iseigaoka, Yahatanishi-ku, Kitakyushu 807-8555, Japan. ${ }^{3}$ Department of Urology, University of Occupational and Environmental Health, Kitakyushu, Japan.

\section{Acknowledgements \\ There is no special mention.}

\section{Compliance with ethical guidelines}

\section{Competing interests}

The authors declare that they have no competing interests.

\section{Consent for publication}

Informed consent was obtained from all individual participants included in the study.

\section{Ethical approval}

This study was approved by the Institutional Review Board of the University of Occupational and Environmental Health.

Received: 9 March 2015 Accepted: 6 July 2015

Published online: 15 July 2015

\section{References}

Abe M, Hiraoka M, Takahashi M, Egawa S, Matsuda C, Onoyama Y et al (1986) Multi-institutional studies on hyperthermia using an 8-MHz radiofrequency capacitive heating device (Thermotron RF-8) in combination with radiation for cancer therapy. Cancer 58:1589-1595

Anscher MS, Samulski TV, Leopold KA, Oleson JR (1992) Phase I/II study of external radio frequency phased array hyperthermia and external beam radiotherapy in the treatment of prostate cancer: technique and results of intraprostatic temperature measurements. Int J Radiat Oncol Biol Phys 24:489-495

Barentsz JO, Richenberg J, Clements R, Choyke P, Verma S, Villeirs G et al (2012) ESUR prostate MR guidelines 2012. Eur Radiol 22:746-757

Boehmer D, Maingon P, Poortmans P, Baron MH, Miralbell R, Remouchamps V et al (2006) Guidelines for primary radiotherapy of patients with prostate cancer. Radiother Oncol 79:259-269

Chang JH, Lim Joon D, Nguyen BT, Hiew CY, Esler S, Angus D et al (2014) MRI scans significantly change target coverage decisions in radical radiotherapy for prostate cancer. J Med Imaging Radiat Oncol 58:237-243

Eggener SE, Yossepowitch O, Serio AM, Vickers AJ, Scardino PT, Eastham JA (2007) Radical prostatectomy shortly after prostate biopsy does not affect operative difficulty or efficacy. Urology 69:1128-1133

Ekici S, Ozen H, Agildere M, Ozkardeş H, Ayhan A, Kendi S (1999) A comparison of transrectal ultrasonography and endorectal magnetic resonance imaging in the local staging of prostatic carcinoma. BJU Int 83:796-800

Engelbrecht MR, Jager GJ, Laheij RJ, Verbeek AL, van Lier HJ, Barentsz JO (2002) Local staging of prostate cancer using magnetic resonance imaging: a meta-analysis. Eur Radiol 12:2294-2302
Futterer JJ, Engelbrecht MR, Jager GJ, Hartman RP, King BF, Hulsbergen-Van de Kaa CA (2007) Prostate cancer: comparison of local staging accuracy of pelvic phased-array coil alone versus integrated endorectal-pelvic phased-array coils. Local staging accuracy of prostate cancer using endorectal coil MR imaging. Eur Radiol 17:1055-1065

Graser A, Heuck A, Sommer B, Massmann J, Scheidler J, Reiser M (2007) Persextant localization and staging of prostate cancer: correlation of imaging findings with whole-mount step section histopathology. AJR Am J Roentgenol 188:84-90

Hayden AJ, Martin JM, Kneebone AB, Lehman M, Wiltshire KL, Skala M (2010) Australian and New Zealand Faculty of Radiation Oncology GenitoUrinary Group: 2010 consensus guidelines for definitive external beam radiotherapy for prostate carcinoma. J Med Imaging Radiat Oncol 54:513-525

Heijmink SW, Futterer JJ, Hambrock T, Takahashi S, Scheenen TW, Huisman $\mathrm{HJ}$ et al (2007) Prostate cancer: body-array versus endorectal coil MR imaging at 3 T_comparison of image quality, localization, and staging performance. Radiology 244:184-195

Jager GJ, Severens JL, Thornbury JR, de La Rosette JJ, Ruijs SH, Barentsz JO (2000) Prostate cancer staging: should MR imaging be used? - A decision analytic approach. Radiology 215:445-451

Kurhanewicz J, Vigneron D, Carroll P, Coakley F (2008) Multiparametric magnetic resonance imaging in prostate cancer: present and future. Curr Opin Urol 18:71-77

Maluta S, Dall'Oglio S, Romano M, Genna M, Oliani C, Pioli F et al (2007) Conformal radiotherapy plus local hyperthermia in patients affected by locally advanced high risk prostate cancer: preliminary results of a prospective phase II study. Int J Hyperthermia 23:451-456

Mullerad M, Hricak H, Wang L, Chen HN, Kattan MW, Scardino PT (2004) Prostate cancer: detection of extracapsular extension by genitourinary and general body radiologists at MR imaging. Radiology 232:140-146

National Comprehensive Cancer Network (2014). Fort Washington, PA. http:// www.nccn.org/. Last accessed 1 Sept 2014

Park BK, Kim B, Kim CK, Choi JY (2007) Comparison of phased-array 3.0-T and endorectal 1.5-T magnetic resonance imaging in the evaluation of local staging accuracy for prostate cancer. J Comput Assist Tomogr 31:534-538

Presti JC Jr, Hricak H, Narayan PA, Shinohara K, White S, Carroll PR (1996) Local staging of prostatic carcinoma: comparison of transrectal sonography and endorectal MR imaging. AJR Am J Roentgenol 166:103-108

Rifkin MD, Zerhouni EA, Gatsonis CA, Quint LE, Paushter DM, Epstein Jl et al (1990) Comparison of magnetic resonance imaging and ultrasonography in staging early prostate cancer. Results of a multi-institutional cooperative trial. N Engl J Med 323:621-626

Roach M 3rd, Hanks G, Thames H Jr, Schellhammer P, Shipley WU, Sokol GH et al (2006) Defining biochemical failure following radiotherapy with or without hormonal therapy in men with clinically localized prostate cancer: recommendations of the RTOG-ASTRO Phoenix Consensus Conference. Int J Radiat Oncol Biol Phys 65:965-974

Rodrigues G, Lukka H, Warde P, Brundage M, Souhami L, Crook J (2013) The prostate cancer risk stratification (ProCaRS) project: recursive partitioning risk stratification analysis. Radiother Oncol 109:204-210

Sala E, Akin O, Moskowitz CS, Eisenberg HF, Kuroiwa K, Ishill NM et al (2006) Endorectal MR imaging in the evaluation of seminal vesicle invasion: diagnostic accuracy and multivariate feature analysis. Radiology 238:929-937

Sciarra A, Barentsz J, Bjartell A, Eastham J, Hricak H, Panebianco V et al (2011) Advances in magnetic resonance imaging: how they are changing the management of prostate cancer. Eur Urol 59:962-977

Sfoungaristos S, Perimenis P (2012) Clinical and pathological parameters predicting extracapsular disease in patients undergoing a radical prostatectomy for clinically localized prostate cancer. Prague Med Rep 113:5-15

Torricelli P, Cinquantini F, Ligabue G, Bianchi G, Sighinolfi P, Romagnoli R (2006) Comparative evaluation between external phased array coil at $3 \mathrm{~T}$ and endorectal coil at 1.5 T: preliminary results. J Comput Assist Tomogr 30:355-361

Turkbey B, Pinto PA, Choyke PL (2009) Imaging techniques for prostate cancer: implications for focal therapy. Nat Rev Urol 6:191-203

Van Vulpen M, De Leeuw AA, Raaymakers BW, Van Moorselaar RJ, Hofman P, Lagendijk JJ et al (2004) Radiotherapy and hyperthermia in the treatment of patients with locally advanced prostate cancer: preliminary results. BJU Int 93:36-41 
Wang L, Mullerad M, Chen HN, Eberhardt SC, Kattan MW, Scardino PT et al (2004) Prostate cancer: incremental value of endorectal MR imaging findings for prediction of extracapsular extension. Radiology 232:133-139
Yu KK, Hricak H, Alagappan R, Chernoff DM, Bacchetti P, Zaloudek CJ (1997) Detection of extracapsular extension of prostate carcinoma with endorectal and phased-array coil MR imaging: multivariate feature analysis. Radiology 202:697-702

\section{Submit your manuscript to a SpringerOpen ${ }^{\odot}$} journal and benefit from:

- Convenient online submission

- Rigorous peer review

- Immediate publication on acceptance

- Open access: articles freely available online

- High visibility within the field

- Retaining the copyright to your article

Submit your next manuscript at $\gg$ springeropen.com 